\title{
New biomarkers of good prognosis in colorectal cancer identified
}

High levels of intratumoral T cells, nuclear STAT1 and MHC class I expression are independently associated with increased survival in patients with colorectal cancer, according to a UK study.

Research suggests that the immune system might be involved in the development and progression of colorectal cancer. Lindy Durrant, lead author of the UK study, explains that she and her team were "interested to see if the immune system, and in particular T cells and the IFN (interferon) $\gamma$ pathway, was operational and influenced survival."

The group screened for key markers of T cells and the IFN- $\gamma$ pathway using tissue microarrays of 462 archived specimens of primary invasive colorectal cancer tissue. Expression of these markers was correlated with patient survival.

Patients whose tumors had a high level of intratumoral $\mathrm{T}$ cells and nuclear STAT1 expression survived for an average 20 months longer than patients with low expression of these markers. Similarly, patients with strong MHC class I expression and a high intratumoral T-cell level survived for an average 26.1 months longer than patients with weak expression of these markers.

The researchers conclude that high levels of intratumoral T cells, nuclear STAT1 and MHC class I can identify patients with an intact immune system who might benefit from immunotherapy, as well as identifying patients for whom this therapy should be avoided. Within the next $2-3$ years the investigators are "planning to trial a new vaccine for colorectal cancer in patients with an intact immune system $(60 \%$ of patients with colorectal cancer)."

\section{Claire Greenhill}

Original article Simpson, J. A. D. et al. Intratumoral T cell infiltration, MHC class I and STAT1 as biomarkers in colorectal cancer. Gut 59, 926-933 (2010) 